Cahiers du Cahiers du monde russe

MONDE RUSSE Russie - Empire russe - Union soviétique et États indépendants

$55 / 3-4 \mid 2014$

Varia

\title{
Wayne Dowler, Russia in 1913
}

\section{Michel Tissier}

\section{OpenEdition \\ Journals}

Édition électronique

URL : http://journals.openedition.org/monderusse/8060

DOI : $10.4000 /$ monderusse. 8060

ISSN : $1777-5388$

Éditeur

Éditions de l'EHESS

Édition imprimée

Date de publication : 1 juillet 2014

Pagination : $397-400$

ISBN : 978-2-7132-2441-6

ISSN : $1252-6576$

\section{Référence électronique}

Michel Tissier, «Wayne Dowler, Russia in 1913 », Cahiers du monde russe [En ligne], 55/3-4 | 2014, mis en ligne le 13 avril 2015, Consulté le 25 septembre 2020. URL : http://journals.openedition.org/ monderusse/8060; DOI : https://doi.org/10.4000/monderusse.8060

Ce document a été généré automatiquement le 25 septembre 2020

〔c École des hautes études en sciences sociales 


\title{
Wayne Dowler, Russia in 1913
}

\author{
Michel Tissier
}

\section{RÉFÉRENCE}

Wayne DOWLER, Russia in 1913, DeKalb : Northern Illinois University Press, 2010, $\mathrm{X}-351 \mathrm{p}$.

1 L'ouvrage de l'historien canadien Wayne Dowler sur la Russie en 1913 peut se lire de plusieurs manières. Il s'agit d'abord d'un livre de synthèse qui tire habilement parti de la riche historiographie consacrée, depuis les années 1980, aux dernières années d'existence du régime impérial, en se fondant principalement sur les travaux en langue anglaise et, dans une moindre mesure, en langue russe. À cet égard, l'ouvrage constitue une variation sur le motif du «sujet-tableau », familier des étudiants, qui permet de dresser un panorama de la situation et des dynamiques à l'œuvre en Russie à la veille de la Première Guerre mondiale. Le résultat est heureusement bien plus intéressant qu'une dissertation, grâce à la connaissance que l'auteur a de l'histoire russe, mais aussi parce qu'il brasse large tout en étant clair et précis. Il ne s'est pas contenté de s'appuyer sur la littérature historique. Il a aussi fait un recours systématique, pour cette année 1913, à deux titres politiquement opposés de la presse russe - moscovite plus précisément -, le journal conservateur Moskovskie vedomosti et le journal proche du parti constitutionnel-démocrate, Russkie vedomosti. On aurait d'ailleurs parfois aimé que l'auteur fasse davantage entendre dans le texte même la voix de ces organes de presse, abondamment mentionnés en notes. Quoi qu'il en soit, son travail, publié en 2010, peut être avantageusement mis en relation, pour les compléter, avec les publications plus nombreuses, récentes et encore à venir, consacrées à la Russie pendant la période de la guerre et de la révolution.

2 De ce premier point de vue, la qualité du livre tient à une structure habile, loin du découpage banal entre vie politique, économie et société, arts et culture, en dépit même de ce que laisse initialement supposer le chapitrage (qui commence par un chapitre sur « Population et économie »). Au contraire, la lecture progresse dans une 
description dense, informative sur chacun de ces différents aspects, mais surtout articulée à une étude des dynamiques affectant la Russie tsariste au début du vingtième siècle. Cette étude s'appuie sur une comparaison récurrente, sans être fastidieuse, avec les autres puissances européennes, ou avec les États-Unis d'Amérique. Le tableau qui en ressort nourrit un argument déjà avancé dans d'autres travaux récents, consistant à rappeler tout ce que la Russie partageait avec les plus grands pays d'Europe, et à mettre en cause les discours - ceux de l'époque comme ceux des historiens - qui martèlent son exceptionnalité. La Russie est montrée comme un "membre de la famille», pour paraphraser le titre d'un ouvrage collectif récent ${ }^{1}$.

3 Au-delà de ce premier niveau de lecture qu'il permet, le livre déroule une interprétation de plus large portée. À travers le déroulé des chapitres suivants «Ordres et classes [sociales] » (chapitre 2), «Intégration sociale et société civile » (3), «L'extension de la société civile » (4), «État et société » (5), « Discours » (6) - c'est la notion de société civile, généralement combinée à celle d'espace public, qui sert de fil directeur à l'auteur. Celui-ci cherche à montrer comment la Russie au début du vingtième siècle traversait, à l'instar des grands pays européens, un processus de consolidation et d'expansion de la "société civile", fondamentalement lié à l'affirmation du marché dans l'économie du pays et dans la vie quotidienne de ses habitants (ce que l'auteur appelle notamment commercial culture, et même commercial popular culture, pour désigner le rôle de ce marché dans la vie des masses, rurales en particulier). La limite est que, selon l'auteur, les conséquences de ce processus se faisaient encore sentir de façon trop timide ou contradictoire dans l'ordre politique, du fait des blocages institutionnels et des divisions internes qui caractérisaient le régime impérial (chapitre 5), mais surtout à cause du poids des idéologies concurrentes du libéralisme, sur la gauche et sur la droite (chapitre 6).

Le moteur matériel et pratique de l'évolution que décrit l'auteur était le capitalisme, et l'agent historique principal en était la « classe moyenne », en train de croître en Russie. Wayne Dowler présente ce groupe central en concédant qu'il restait fragile en 1913, mais en soulignant la force de ses aspirations et des effets d'entraînement dont il était capable. Sa vision prend ainsi le contrepied des interprétations qui soulignent l'absence (supposée dommageable) d'une « bourgeoisie » dans le développement russe. S'il admet le constat d'une "fragmentation » de cette nouvelle classe moyenne russe, l'auteur n'y voit pas une spécificité de la Russie de l'époque dans le paysage des «sociétés bourgeoises " européennes ni un élément susceptible d'infirmer sa thèse d'une moyennisation de la société russe. Il affirme au contraire le pouvoir mobilisateur du modèle bourgeois sur de vastes parties de la population de l'empire, y compris la population ouvrière des capitales, Saint-Pétersbourg et Moscou.

5 En réfléchissant à la place que tient cette interprétation dans le propos de l'auteur, on voit bien que le livre ouvre à une seconde lecture qu'il a clairement encouragée, dans sa préface notamment, mais qu'il s'est gardé de développer dans le fil de l'ouvrage. Si cela n'avait pas contredit le projet de faire une synthèse sur 1913, l'auteur aurait peut-être cédé à la tentation d'écrire un livre pour affronter plus directement la question du rôle joué par la période de la guerre et, in fine, celle des causes de la chute du régime tsariste et des révolutions de 1917. Le fait que la question ne soit pas traitée directement ne signifie pas que l'auteur ne l'a pas résolue d'une certaine façon, ou qu'il n'a pas suggéré des pistes pour la résoudre. Ainsi, la justification de son projet, dans la préface, tient à l'ambition de montrer l'éventail des possibles ouverts à l'histoire russe 
en cette année 1913 et, partant, au refus de faire une téléologie de la révolution. À la déclaration de méthode fournie par la préface ( A detailed study of a society in a single year not only reveals its complexity, but also the potential that complexity contained", p. VII), répond la conclusion spécifique à laquelle l'auteur a abouti : « If Russia was still far from becoming a liberal capitalist democracy in 1913, it was even farther from socialist revolution. Severe stresses and tensions remained but the clear trend before the war was toward cooperation and integration» (p. 279). Le livre n'entre pas dans le genre de l'histoire contrefactuelle, mais on voit bien que les conclusions de l'auteur tendent dans cette direction, d'ailleurs naturelle à la démarche historique, mais de façon sans doute plus affirmée qu'à l'ordinaire².

6 C'est donc aussi naturellement qu'il faut chercher à se prononcer sur sa vision du potentiel transformateur, pour la Russie de 1913, du «discours libéral de la classe moyenne » (p. 244-251, 279), dans l'hypothèse où la guerre ne serait pas survenue. Un premier point peut nourrir la discussion : c'est la façon dont l'auteur traite la question de la chronologie. D'une part, de façon très intéressante, il remet en cause à plusieurs reprises la dénomination floue - mais très présente dans les études historiques anglophones et au-delà - de " late Imperial Russia » (p. VII, 17, 45). En déconstruisant cette période fourre-tout pour mieux s'intéresser à 1913, Wayne Dowler insiste sur les caractéristiques du moment que l'on peut appeler l'après-1905, avec ses désillusions, ses remises en cause et ses nouveaux départs. Mais, d'autre part, reconnaitre les spécificités de ce moment et les évolutions rapides qu'il révélait ne permet pas de décider si elles avaient le potentiel pour contrebalancer les effets de relations plus anciennement constituées. Il s'agit non seulement de la question de la solidité de l'ordre institutionnel tsariste, mais aussi des antagonismes existant dans les campagnes, ou encore dans les provinces allogènes dominées par les Russes, problèmes que discute l'auteur au chapitre 4 . Si sa réponse implicite à la question du pourquoi des révolutions paraît être celle de l'accident de parcours, le fait de singulariser positivement la période 1906-1914 ne suffit pas à en prouver la validité. Il faudrait comparer cette période aussi bien avec l'héritage antérieur qu'avec les conséquences de la guerre même.

7 D’autres présupposés peuvent également être discutés, comme le lien postulé par l'auteur entre le développement d'une économie capitaliste, la culture du marché et l'essor d'un esprit démocratique libéral. Ce lien ne paraît pas très convaincant si l'on retient des exemples pris dans d'autres pays d'Europe à la même époque, ou même si l'on élargit la perspective à une période plus récente, ailleurs dans le monde. L'analyse développée par l'auteur est ici circonscrite par une conception univoque du "libéralisme», utilisée comme une norme, qui plus est d'origine intrinsèquement occidentale (p.244-251). Cette façon de juger l'histoire idéologique russe semble contredire le projet de reconnaître la Russie comme un "membre de la famille». Certains rétorqueront - la chose est facile - que les grands penseurs du libéralisme ne furent pas russes... C'est pourtant un peu court, si l'on veut s'intéresser au libéralisme non pas comme à un corpus constitué et hiérarchisé, mais comme à une pensée élaborée, manipulée et appropriée de bien des façons. Ce fut en particulier le cas en Russie au début du vingtième siècle, pendant et après 1905, mais c'est aussi vrai pour l'histoire moderne des pays occidentaux qui servent généralement de points de référence. Wayne Dowler oppose, d'une part, l'omniprésence de discours et idéologies anticapitalistes, antilibérales et, au fond, antidémocratiques (portés, d'après lui, par toutes les forces politiques de gauche et de droite, face à l'évolution économique, 
sociale et même culturelle du pays) et, d'autre part, la faiblesse du discours libéral de la classe moyenne, alors que l'essor de cette dernière est le phénomène majeur qu'il repère. Ce paradoxe est moins une tension interne à la Russie de 1913 qu'un effet d'optique lié aux choix de l'historien. Toute la «complexité » de ce moment n'a donc pas été traitée de façon équivalente par Wayne Dowler. Son ouvrage constitue en tout cas une synthèse réussie et stimulante, parce qu'il a aussi fait, mezza-voce, le pari de l'analyse.

\section{NOTES}

1. Susan McCaffray, Michael Melancon, eds., Russia in the European Context, 1789-1914: A Member of the Family, New York : Palgrave MacMillan, 2005.

2. Quentin Deluermoz, Pierre Singaravélou, «Explorer le champ des possibles: approches contrefactuelles et futurs non advenus en histoire ", Revue d'histoire moderne et contemporaine, 59, 3 (2012), p. 70-94.

\section{AUTEURS}

\section{MICHEL TISSIER}

Université Rennes 2 - CERHIO (UMR 6258 CNRS) 